\title{
Towards the Raychaudhuri Equation Beyond General Relativity
}

\author{
Daniel J Burger ${ }^{*}$ and Nathan Moynihan ${ }^{\dagger}$ \\ Department of Applied Mathematics and Mathematics, \\ University of Cape Town, \\ Cape Town, South Africa \\ Saurya Das $\ddagger$ \\ Department of Physics and Astronomy, \\ University of Lethbridge, 4401 University Drive, \\ Lethbridge, Alberta T1K 3M4, Canada \\ S. Shajidul Haque $\$$ \\ Department of Physics, \\ University of Windsor, \\ Windsor, Ontario N9A 0C5, Canada \\ Bret Underwood \\ Department of Physics, \\ Pacific Lutheran University, \\ Tacoma, WA 98447 \\ (Dated: July 9, 2018)
}

\begin{abstract}
In General Relativity, gravity is universally attractive, a feature embodied by the Raychaudhuri equation which requires that the expansion of a congruence of geodesics is always non-increasing, as long as matter obeys the strong or weak energy conditions. This behavior of geodesics is an important ingredient in general proofs of singularity theorems, which show that many spacetimes are singular in the sense of being geodesically incomplete and suggest that General Relativity is itself incomplete. It is possible that alternative theories of gravity, which reduce to General Relativity in some limit, can resolve these singularities, so it is of interest to consider how the behavior of geodesics is modified in these frameworks. We compute the leading corrections to the Raychaudhuri equation for the expansion due to models in string theory, braneworld gravity, $f(R)$ theories, and Loop Quantum Cosmology, for cosmological and black hole backgrounds, and show that while in most cases geodesic convergence is reinforced, in a few cases terms representing repulsion arise, weakening geodesic convergence and thereby the conclusions of the singularity theorems.
\end{abstract}

\section{INTRODUCTION}

General Relativity (GR) is widely expected to be an incomplete theory of dynamical spacetime. One reason for this is that GR famously predicts its own demise through the existence of singularities, as demonstrated by the singularity theorems [1, 2]. An essential physical ingredient of the singularity theorems is that gravity is attractive, so that congruences of convergent timelike and null geodesics develop singularities in finite proper (affine) time. More specifically, geodesic congruences with timelike $u^{M}(\tau)$ and null $n^{M}(\lambda)$ tangent vector fields are characterized by their expansion $\theta \equiv \nabla_{M} u^{M}, \hat{\theta} \equiv \nabla_{M} n^{M}$, respectively, which satisfy the Raychaudhuri equations [3]

$$
\begin{aligned}
& \frac{d \theta}{d \tau}=-\frac{\theta^{2}}{D-1}-R_{M N} u^{M} u^{N}+\ldots \quad \text { (timelike), } \\
& \frac{d \hat{\theta}}{d \lambda}=-\frac{\hat{\theta}^{2}}{D-2}-R_{M N} n^{M} n^{N}+\ldots \quad \text { (null) }
\end{aligned}
$$

for spacetime dimension $D$, where the additional $+\ldots$ terms are non-positive; see Appendix $\mathrm{A}$ for details.

\footnotetext{
*Electronic address: burgerj.daan@gmail.com

$\dagger$ Electronic address: nathantmoynihan@gmail.com

‡Electronic address: saurya.das@uleth.ca

$\S$ Electronic address: shajid.haque@uwindsor.ca

IElectronic address: underwbj@plu.edu
} 
For the timelike Raychaudhuri equation, if the so-called "convergence condition"

$$
R_{M N} u^{M} u^{N} \geq 0
$$

holds, then the expansion of a congruence of geodesics is non-increasing. Specifically, an initially converging congruence $\theta_{i}<0$ develops a singularity $\theta \rightarrow-\infty$ in finite proper time $\tau \sim\left|\theta_{i}\right|^{-1}$. A similar singularity $\hat{\theta} \rightarrow-\infty$ develops for null convergences in finite affine time $\lambda \sim\left|\hat{\theta}_{i}\right|^{-1}$ if the null convergence condition $R_{M N} n^{M} n^{N} \geq 0$ is satisfied. These singularities in the expansion don't necessarily imply a pathology of spacetime themselves; such caustics appear in Minkowski spacetime, for example [4. However, the existence of strictly non-positive contributions to the right-hand side of Eq.(1), when combined with other global conditions on the spacetime manifold, form the basis for the general existence of singularities in cosmological and black hole spacetimes [1, 2].

Within GR it is possible to convert the convergence conditions into energy conditions on the types of matter. We can use the (trace-reversed) Einstein equation

$$
R_{M N}=\kappa_{D}^{2}\left(T_{M N}-\frac{1}{D-2} g_{M N} T_{M}^{M}\right)
$$

(where $\kappa_{D}^{2} \equiv 16 \pi G_{D}, G_{D}$ being the $D$-dimensional Newton's constant) to substitute in Eq. (1)

$$
\begin{array}{ll}
\frac{d \theta}{d \tau}=-\kappa_{D}^{2}\left(T_{M N}-\frac{1}{D-2} g_{M N} T_{M}^{M}\right) u^{M} u^{N}+\ldots & \text { (timelike) } \\
\frac{d \hat{\theta}}{d \lambda}=-\kappa_{D}^{2} T_{M N} n^{M} n^{N}+\ldots & \text { (null) }
\end{array}
$$

where the $+\ldots$ terms are again strictly non-positive terms. Thus, all of the terms on the right-hand side of (4) are non-positive provided that matter satisfies the respective energy conditions

$$
\begin{aligned}
\left(T_{M N}-\frac{1}{D-2} g_{M N} T_{M}^{M}\right) u^{M} u^{N} & \geq 0 & & \text { Strong Energy Condition, } \\
T_{M N} n^{M} n^{N} & \geq 0 & & \text { Null Energy Condition. }
\end{aligned}
$$

For an isotropic perfect-fluid energy momentum tensor with energy density $\rho$ and pressure $p$, these conditions translate into

$$
\begin{aligned}
\rho+\frac{D-1}{D-3} p \geq 0 & \text { Strong Energy Condition } \\
\rho+p \geq 0 & \text { Null Energy Condition. }
\end{aligned}
$$

Most known classical matter obeys both the null and strong energy conditions, while vacuum energy $p=-\rho$ violates the strong energy condition but still saturates the null energy condition. It may possible to violate the null energy condition with exotic forms of matter [5, 6], non-minimal coupling [7, 8], or quantum gravity effects [9], though these approaches often face challenges that we will not explore further here.

However, since we expect corrections to GR of some form, we do not expect the Einstein equations (3) to always hold. Corrections to Einstein's equations may make it possible to violate the convergence conditions $R_{M N} u^{M} u^{N}<0$ and/or $R_{M N} n^{M} n^{N}<0$ without violating the energy conditions (5). In particular, many corrections to GR appear perturbatively in the form

$$
R_{M N}=\kappa_{D}^{2}\left(T_{M N}-\frac{1}{D-2} g_{M N} T_{M}^{M}\right)+\lambda H_{M N},
$$

where $\lambda$ controls the strength of the corrections and $H_{M N}$ is a tensor that contains contributions from the metric, curvature, energy-momentum tensor, or additional fields. The additional term in Eq.77) in turn shows up as an additional term on the right-hand side of the Raychaudhuri equation

$$
\begin{array}{ll}
\frac{d \theta}{d \tau}=-\kappa_{D}^{2}\left(T_{M N}-\frac{1}{D-2} g_{M N} T_{M}^{M}\right) u^{M} u^{N}+\lambda H_{M N} u^{M} u^{N}+\ldots & \text { (timelike) } \\
\frac{d \hat{\theta}}{d \lambda}=-\kappa_{D}^{2} T_{M N} n^{M} n^{N}+\lambda H_{M N} n^{M} n^{N}+\ldots & \text { (null) }
\end{array}
$$


where it may in principle contribute with any sign. In this paper, we will examine corrections to the Raychaudhuri equations from four frameworks for corrections to GR: string theory, braneworld gravity, $f(R)$ theories, and Loop Quantum Cosmology, for cosmological (and in some cases black hole) backgrounds (see also [10, 11] for a similar analysis in infinite derivative gravity).

While we are motivated by the existence of singularities in these backgrounds, and the promise of these alternatives to GR for resolving the singularities, we will not attempt to prove the absence of singularities in this paper. Indeed, a significant amount of work has shown that finding realistic singularity-free spacetimes under computational control is quite challenging, and we expect that true singularity resolution will require physics beyond the perturbative approach of (7). Instead, we aim for a more modest goal, that of finding corrections to GR that lead to potentially positive terms on the right-hand side of the Raychaudhuri equation Eq.(1) for timelike and null geodesics - a necessary, but far from sufficient, condition for ultimately resolving spacetime singularities. If indeed such positive terms are found (as we shall see for a handful of cases), further analysis would be required to see, whether: (a) a generalization of the singularity theorems holds for these cases, or (b) another criterion for singularity (in lieu of geodesic incompleteness) can be applied. If none of the above holds, one would be forced to conclude that these spacetimes are indeed nonsingular. Such an analysis is beyond the scope of this work, however.

In Section III we outline a general set of corrections to GR from string theory in $D$ dimensions, and compute the corrections to the Raychaudhuri equation for black hole and cosmological spacetimes. In Section III we compute the corrections to the 4-dimensional induced Einstein equation for the braneworld scenario in a cosmological spacetime. In Section IV] we compute the corrections to the Einstein equation for so-called $f(R)$ theories, and determine the form of the corrections in a cosmological background. In Section $\mathrm{V}$ we compute the corrections to the Raychaudhuri equation in a cosmological background from Loop Quantum Gravity. In Section VI we conclude with some comments on the potential for theories beyond GR to resolve singularities.

\section{STRING THEORY AND GAUSS-BONNET CORRECTIONS}

In string theory, higher-order $\alpha^{\prime}$ corrections induce corrections to the action and, correspondingly, to the Einstein Equations. In particular, the corrected action at leading order in $\alpha^{\prime}$ for bosonic, heterotic, type IIA/IIB takes the form $[12$ 14]

$$
S=\frac{1}{\kappa_{D}^{2}} \int d^{D} x \sqrt{-g} e^{-2 \phi}\left[R_{D}+4(\partial \phi)^{2}+\lambda R_{M N P Q} R^{M N P Q}+\mathcal{O}\left(\alpha^{\prime 2}\right)\right]
$$

where we are ignoring the antisymmetric rank two tensor $B_{M N}, \phi$ is the dilaton, $R_{M N P Q}$ is the $D$-dimensional Riemann tensor, and where ${ }^{1}$

$$
\lambda= \begin{cases}\frac{1}{2} \alpha^{\prime} & \text { for bosonic strings } \\ \frac{1}{4} \alpha^{\prime} & \text { for heterotic strings } \\ 0 & \text { for supersymmetric strings (IIA/IIB) }\end{cases}
$$

Unfortunately, (9) contains higher derivative terms in its equation of motion. However, it turns out that $(9)$ is ambiguous up to field redefinitions of the fields $g_{\mu \nu}, \phi$ to next order in $\alpha^{\prime}$. It is possible to use these field redefinitions to remove the higher order terms in the equations of motion ${ }^{2}$, giving rise to the action

$$
\begin{aligned}
S_{\text {mod }}=\frac{1}{\kappa_{D}^{2}} \int d^{D} x \sqrt{-g} e^{-2 \phi} & \left\{R_{D}+4(\partial \phi)^{2}+\frac{1}{2} \lambda\left[R_{G B}^{2}+16\left(R^{M N}-g^{M N} R_{D}\right) \partial_{M} \phi \partial_{N} \phi\right.\right. \\
& \left.\left.-16 \nabla^{2} \phi(\partial \phi)^{2}+16(\partial \phi)^{4}\right]+\mathcal{O}\left(\alpha^{\prime 2}\right)\right\}+\mathcal{L}_{m}
\end{aligned}
$$

where we have allowed for additional matter (including potentially a $D$-dimensional cosmological constant) through $\mathcal{L}_{m}$, and $R_{G B}^{2}$ is the 2 nd order Gauss-Bonnet combination

$$
R_{G B}^{2}=R_{M N P Q} R^{M N P Q}-4 R_{M N} R^{M N}+R_{D}^{2} .
$$

\footnotetext{
1 The leading $\alpha^{\prime}$ corrections for type II theories occurs first at $\mathcal{O}\left(\alpha^{\prime 3}\right)$ [15].

${ }^{2}$ See the discussion in [13, 14, 16].
} 
Setting the dilaton to a constant, we are left with the usual Ricci curvature term and the quadratic Gauss-Bonnet term in our action

$$
S_{E G B}=\frac{1}{\kappa_{D}^{2}} \int d^{D} x \sqrt{-g}\left[R_{D}+\frac{1}{2} \lambda R_{G B}^{2}\right]+\mathcal{L}_{m}
$$

We will refer to this as the Einstein-Gauss-Bonnet (EGB) gravity action. The resulting equations of motion are

$$
R_{M N}-\frac{1}{2} g_{M N} R_{D}=\kappa_{D}^{2} T_{M N}+\frac{1}{2} \lambda \hat{H}_{M N}
$$

where

$$
\hat{H}_{M N}=\frac{g_{M N}}{2} R_{G B}^{2}-2 R_{D} R_{M N}+4 R_{M A} R_{N}^{A}+4 R^{A B} R_{A M B N}-2 R_{M A B C} R_{N}^{A B C} .
$$

In order to put 14 in the appropriate form relevant for use the Raychaudhuri equation ${ }^{3}$, we will trace-reverse, giving

$$
R_{M N}=\kappa_{D}^{2}\left(T_{M N}-\frac{g_{M N}}{D-2} T_{M}^{M}\right)+\frac{\lambda}{2} H_{M N},
$$

where now

$$
H_{M N}=\frac{g_{M N}}{D-2} R_{G B}^{2}-2 R_{D} R_{M N}+4 R_{M A} R_{N}^{A}+4 R^{A B} R_{A M B N}-2 R_{M A B C} R_{N}^{A B C} .
$$

More generally, the terms above are just the leading terms of the more generic Lanczos-Lovelock extensions of gravity [18, 19] (see [20] for a review). The Lanczos-Lovelock extensions include the set of additional terms that can be added to the gravitational action and still lead to 2nd order equations of motion. In 4 spacetime dimensions, it can be shown that the Ricci scalar and cosmological constant are the only non-topological terms that can be added. In particular, the correction term in 11 is purely topological in 4-dimensions (it is the Euler characteristic $\chi$ of the spacetime), not contributing to the equation of motion. For higher dimensions $D>4$, there is a finite series of additional terms, increasing in powers of $R, R_{M N}, R_{N P Q}^{M}$ (terminating at some order for a given $D$ ). We will just focus on this leading order term for now, but will keep in mind that there can be additional corrections to consider.

In order to determine the form of these corrections it is necessary to calculate $H_{M N}$ for specific backgrounds. For simplicity, we will restrict ourselves to corrections to black hole and cosmological backgrounds; in the subsections that follow, we will examine the corrections for these backgrounds in more detail.

\section{A. Black Holes}

The Gauss-Bonnet correction terms (16) appear as part of a pertubative series of corrections, so we expect solutions for some given matter content to also be described by a pertubative series in $\lambda: g_{M N}(\lambda)=g_{M N}^{(0)}+\lambda g_{M N}^{(1)}+\ldots$, where $g_{M N}^{(0)}$ is the uncorrected General Relativity solution, $g_{M N}^{(1)}$ is the first-order correction, and so on. It is remarkable that exact solutions $g_{M N}(\lambda)$ of Einstein-Gauss-Bonnet gravity for black hole backgrounds are known to all orders in $\lambda$ [21 23]. However, since we expect the Gauss-Bonnet corrections to be only the first term in a series of corrections arising from string theory, we cannot trust these exact solutions beyond $\mathcal{O}(\lambda)$. One can therefore consider the effects of the Gauss-Bonnet correction terms on the Raychaudhuri equations up to $\mathcal{O}(\lambda)$ for black hole backgrounds in two ways:

1. Evaluate $R_{M N} u^{M} u^{N} \sim \frac{\lambda}{2} H\left[g^{(0)}\right]_{M N} u^{M} u^{N}$ on the uncorrected Schwarzschild black hole metric.

2. Evaluate $R[g(\lambda)]_{M N} u^{M} u^{N}$ on the known exact metric $g_{M N}(\lambda)$ for black hole backgrounds, then expand the result to $\mathcal{O}(\lambda)$.

(with similar expressions for the null Raychaudhuri equation). While both approaches should give identical results, up to $\mathcal{O}(\lambda)$, we will consider and compare both approaches for completeness.

\footnotetext{
${ }^{3}$ See [17] for an alternative method of deriving a generalized Raychaudhuri equation for EGB gravity.
} 


\section{Perturbative Black Hole Corrections}

Since we are considering actions with a constant dilaton and zero form fields, we will restrict ourselves to pure gravity solutions. We thus first begin by considering the perturbative correction terms $H_{M N}$ to a $D$-dimensional black hole solution of the vacuum Einstein equations

$$
d s^{2}=-f(r) d t^{2}+\frac{d r^{2}}{f(r)}+r^{2} d \Omega_{D-2}^{2},
$$

where $d \Omega_{D-2}^{2}=\hat{g}_{i j} d \theta^{i} d \theta^{j}$ is the metric of a $(D-2)$-dimensional constant curvature manifold with unit radius (such as a sphere). The zeroth order black hole solution takes the form

$$
f(r)=1-\frac{\mu}{r^{D-3}}
$$

where $\mu$ is related to the mass $M$ of the black hole by

$$
M=\frac{(D-2) A_{D-2}}{2 \kappa_{D}^{2}} \mu
$$

and $A_{D-2}$ is the area of a unit $(D-2)$ sphere. Note that for $D=4$ we have $\mu=\kappa_{D}^{2} M / 4 \pi$.

While the Ricci tensor and scalar vanish $R_{M N}=0=R_{D}$, as is expected for a $D$-dimensional Schwarzschild background, the Riemann tensor does not, and gives the only non-zero contribution to the Gauss-Bonnet scalar

$$
R_{G B}^{2}=R_{A B C D} R^{A B C D}=(D-3)(D-2)^{2}(D-1) \frac{\mu^{2}}{r^{2 D-2}},
$$

and corrections to the Einstein equation,

$$
H_{M N}=\frac{g_{M N}}{D-2}\left[R_{A B C D} R^{A B C D}\right]-2 R_{M A B C} R_{N}^{A B C}
$$

We are primarily concerned with the correction terms in the $(t t)$ and $(r r)$ directions,

$$
\begin{aligned}
& H_{t t}=\frac{f(r) \mu^{2}}{r^{2 D-2}}(D-4)(D-3)(D-2)(D-1) \\
& H_{r r}=-\frac{1}{f(r)} \frac{\mu^{2}}{r^{2 D-2}}(D-4)(D-3)(D-2)(D-1) .
\end{aligned}
$$

Note that these correction terms vanish identically $H_{M N}=0$ for $D \leq 4$, which is what we expect since the GaussBonnet correction is purely topological for $D \leq 4$, and only acts as a dynamical correction for $D>4$.

We now consider a radial affine null tangent vector

$$
n^{M}=\left(\frac{1}{f(r)}, \pm 1, \overrightarrow{0}\right)
$$

where \pm 1 corresponds to radially outgoing/ingoing. The null Raychaudhuri equation takes the form

$$
\frac{d \hat{\theta}}{d \lambda}=\frac{-\hat{\theta}^{2}}{D-2}-|\hat{\sigma}|^{2}-R_{M N} n^{M} n^{N}
$$

Our corrections due to the Gauss-Bonnet term appear on the right hand side, as (recall that $T_{M N}=0$ )

$$
R_{M N} n^{M} n^{N}=\frac{\lambda}{2} H_{M N} n^{M} n^{N}=\frac{\lambda}{2} H_{t t} n^{t} n^{t}+\frac{\lambda}{2} H_{r r} n^{r} n^{r}=0 .
$$

Remarkably, the Gauss-Bonnet corrections vanish identically everywhere for null rays, implying that the null Raychaudhuri equation for black holes is uncorrected to leading order in $\lambda$.

Finally, consider a timelike geodesic described by the tangent vector

$$
u^{M}=\left(\frac{1}{f(r)},-\left(\frac{\mu}{r^{D-3}}\right)^{1 / 2}, \overrightarrow{0}\right) .
$$


The timelike Raychaudhuri equation takes the form

$$
\frac{d \theta}{d \tau}=-\frac{\theta^{2}}{D-1}-|\sigma|^{2}-R_{M N} u^{M} u^{N},
$$

where again the corrections to the Raychaudhuri equation due to the Gauss-Bonnet corrections come from the last term

$$
R_{M N} u^{M} u^{N}=\frac{\lambda}{2} H_{M N} u^{M} u^{N}=\frac{\lambda}{2} H_{t t} u^{t} u^{t}+\frac{\lambda}{2} H_{r r} u^{r} u^{r}=\frac{\lambda(D-4)(D-3)(D-2)(D-1)}{2} \frac{\mu^{2}}{r^{2 D-2}} .
$$

There are a few things to notice about this correction term. First, it does not seem to suffer from any pathologies due to the coordinate singularity of our coordinate system at the horizon; thus, we expect it to be valid throughout the entire spacetime (although the coordinate $r$ will require careful interpretation). Second, notice that the corrections are manifestly positive, thus the corrections make the caustic/conjugate point at the location of the putative singularity at $r=0$ worse, not better!

We have shown that the perturbative EGB corrections to the Einstein equations do not provide divergence terms in either the null or timelike Raychaudhuri equations; in fact, convergence is strengthened in the timelike case. These results above are not particularly surprising since it is known that black hole solutions in Einstein-Gauss-Bonnet gravity still posses singularities [21 23], as we will now examine in more detail.

\section{Exact Black Hole Solutions}

In the previous section we considered how the Gauss-Bonnet curvature squared terms lead to perturbative corrections to the Raychaudhuri equation for pure-Einstein gravity black hole solutions. However, exact black hole solutions for Einstein-Gauss-Bonnet gravity are well-known [21 23. (see also [12), so it is also possible to calculate the right-hand-side of the Raychaudhuri equation for these fully backreacted solutions.

First, let us review the known black hole solutions of $D$-dimensional Einstein-Gauss-Bonnet gravity [21 23]. As before, we will work with the general spherically symmetric metric

$$
d s_{D}^{2}=-f(r) d t^{2}+\frac{d r^{2}}{f(r)}+r^{2} d \Omega_{D-2}^{2} .
$$

Solutions to the Einstein-Gauss-Bonnet equations of motion (16) are [21] (see also [22, 23])

$$
f(r)=1+\frac{r^{2}}{\hat{\lambda}}+\sigma \frac{r^{2}}{\hat{\lambda}} \sqrt{1+\frac{2 \hat{\lambda} \mu}{r^{D-1}}}
$$

where $\sigma= \pm 1$ labels different branches of solutions, and we have defined $\hat{\lambda}=(D-4)(D-3) \lambda$ for convenience.

Consider first the $\sigma=-1$ branch. To lowest order in $\lambda$, the metric is simply that of a Schwarzschild black hole, and is asymptotically flat as $r \rightarrow \infty$, reducing to the usual Einstein gravity solution. For this reason, this is usually called the "Einstein branch." In contrast, the $\sigma=+1$ branch, often called the "Gauss-Bonnet branch," has a non-trivial vacuum structure for $\mu=0$

$$
\left.f(r)\right|_{\mu=0}=1+\frac{2 r^{2}}{\hat{\lambda}}
$$

corresponding to anti-deSitter space for $\lambda>0$ with a large negative cosmological constant $\Lambda_{e f f} \sim-\hat{\lambda}^{-1}$ (or deSitter space for $\lambda<0$ ). For non-zero mass, the metric in this branch resembles that of Schwarzschild-anti-deSitter space with a negative mass

$$
f(r) \approx 1+\frac{\mu}{r^{D-3}}+\frac{2 r^{2}}{\hat{\lambda}}+\mathcal{O}\left(\lambda^{2}\right)
$$

The analysis of the stability of this branch requires some care (see 21, 24]). Note that since the curvature scale of the corresponding AdS space is non-perturbative in $\lambda$, it is doubtful that we can trust these solutions as solutions to perturbative string theory. Further, for $\lambda>0$ there is a naked singularity at the origin [21. For these reasons, we will restrict our analysis to the Einstein branch $\sigma=-1$. 
Despite the curvature squared term in EGB gravity, the Einstein branch solutions (3132) still have a curvature singularity at the origin. This singularity is surrounded by a horizon located at $r_{h}$, given by the roots of the polynomial [21]

$$
\hat{\lambda} r_{h}^{D-5}+2 r_{h}^{D-3}=2 \mu \text {. }
$$

For $D>5$, this horizon always exists; however, for $D=5$, the existence of the horizon is guaranteed only for $\mu>\hat{\lambda} / 2$; thus, for "microscopic" black holes, even the EGB solutions posses a naked singularity. Since the exact EGB black hole solutions still posses a curvature singularity at the origin, we are not particularly surprised by our perturbative result from the previous subsection indicating that the corrections to the null and timelike Raychaudhuri equations do not allow for terms that could prevent the formation of conjugate points.

Even though we have the exact EGB solutions, which do possess a curvature singularity, in hand, let us nevertheless compute the contribution of EGB gravity to the right-hand side of the Raychaudhuri equation to explore the way in which the EGB corrections can affect the formation of conjugate points.

Note that since the solutions $(31 \mid 32)$ are exact solutions to the corrected Einstein equations

$$
R_{M N}-\frac{1}{2} g_{M N} R=\kappa_{D}^{2} T_{M N}+\frac{1}{2} \lambda H_{M N},
$$

we can compute the curvature term in the null (timelike) Raychaudhuri equation $R_{M N} N^{M} N^{N}\left(R_{M N} u^{M} u^{N}\right.$ respectively) directly, without needing to compute the quadratic curvature terms.

For the metric (31), we have

$$
\begin{aligned}
& R_{t t}=\frac{1}{2} f(r)\left(f^{\prime \prime}(r)+\frac{(D-2)}{r} f(r)\right) \\
& R_{r r}=-\frac{1}{2} \frac{1}{f(r)}\left(f^{\prime \prime}(r)+\frac{(D-2)}{r} f(r)\right),
\end{aligned}
$$

where a prime' denotes a derivative with respect to $r$. Note that these vanish for a Schwarzschild solution $f(r)=$ $1-\frac{\mu}{r^{D-3}}$, as expected.

A radial affine null vector has the same form as in the Schwarzschild case

$$
n^{M}=\left(\frac{1}{f(r)}, \pm 1, \overrightarrow{0}\right)
$$

where now $f(r)$ refers to the EGB corrected form 32 . The curvature term in the Raychaudhuri equation is then

$$
R_{M N} n^{M} n^{N}=R_{t t}\left(n^{t}\right)^{2}+R_{r r}\left(n^{r}\right)^{2}=0,
$$

which again vanishes identically, as we found previously in the perturbative case. It is important to note that the vanishing result is independent of the precise functional form of $f(r)$, and only requires the generic structure of the metric (31).

The geodesic timelike null vector for the metric (31) takes the form

$$
u^{M}=\left(\frac{1}{f(r)}, \pm \sqrt{1-f(r)}, \overrightarrow{0}\right),
$$

where we are assuming $f(r)<1$, which is the case for the Einstein branch of solutions. The curvature term in the Raychaudhuri equation then takes the form

$$
R_{M N} u^{M} u^{N}=R_{t t}\left(u^{t}\right)^{2}+R_{r r}\left(u^{r}\right)^{2}=\frac{1}{2}\left(f^{\prime \prime}(r)+\frac{(D-2)}{r} f^{\prime}(r)\right) .
$$

The general expression for $f(r)$ given in 32 is not particularly illuminating; however, expanding the solution in powers of $\lambda$, we obtain

$$
R_{M N} u^{M} u^{N} \approx \frac{\lambda(D-4)(D-3)(D-2)(D-1) \mu^{2}}{4 r^{2 D-2}}+\mathcal{O}\left(\lambda^{2}\right),
$$

matching our perturbative result $(30)$, as expected. 
As in the perturbative analysis, we see that the EGB corrections to the Schwarzschild black hole background give rise to either vanishing or convergent contributions to the null and timelike Raychaudhuri equations. Thus, the EGB corrections themselves, despite being quadratic in curvature, do not alleviate convergence that leads to conjugate points and singularities in these spaces.

We have restricted ourself to spherically symmetric black hole solutions for simplicity; however, since we have not found an improvement in the convergence behavior, we do not expect that deviations from spherical symmetry are likely to produce qualitatively different results.

Black hole backgrounds are not the only spacetimes of interest for the study of singularities and the Raychaudhuri equation. In the next subsection, we will explore the application of EGB gravity to cosmological spacetimes.

\section{B. Cosmology}

Let's examine the EGB corrections 17 for a $D$-dimensional cosmological background ${ }^{4}$

$$
d s^{2}=-d t^{2}+a(t)^{2}\left(d r^{2}+r^{2} \hat{d}_{D-2}^{2}\right)
$$

where $\hat{d}_{D-2}^{2}=\hat{g}_{i j} d \theta^{i} d \theta^{j}$ is the metric of a $(D-2)$-dimensional sphere. Our matter will consist of a $D$-dimensional perfect fluid

$$
T_{M N}=(\rho+p) u_{M} u_{N}+p g_{M N},
$$

where $\rho, p$ are the energy density and pressure of the fluid, respectively.

It is straightforward to compute the Gauss-Bonnet scalar

$$
\begin{aligned}
R_{G B}^{2} & =R_{A B C D} R^{A B C D}-4 R_{M N} R^{M N}+\left(R_{D}\right)^{2} \\
& =4 \frac{\ddot{a}}{a} \frac{\dot{a}^{2}}{a^{2}}(D-3)(D-2)(D-1)+\frac{\dot{a}^{4}}{a^{4}}(D-4)(D-3)(D-2)(D-1),
\end{aligned}
$$

and the correction terms 17

$$
\begin{aligned}
& H_{t t}=2 \frac{\ddot{a}}{a} \frac{\dot{a}^{2}}{a^{2}}(D-4)(D-3)(D-1)-(D-4)(D-3)(D-1) \frac{\dot{a}^{4}}{a^{4}} ; \\
& H_{r r}=-2 \frac{\ddot{a} \dot{a}^{2}}{a}(D-4)(D-3)-\frac{\dot{a}^{4}}{a^{2}}(D-4)(D-3)^{2} .
\end{aligned}
$$

Notice that these corrections vanish identically for $D \leq 4$, as expected since for lower dimensions the EGB terms are topological and don't contributed to the equations of motion.

From the radial affine null tangent vector

$$
n^{M}=\left(\frac{1}{a(t)}, \pm \frac{1}{a(t)^{2}}, \overrightarrow{0}\right)
$$

where again \pm refers to radially outgoing/ingoing rays, we can compute the corrections to the null Raychaudhuri equation

$$
\begin{aligned}
R_{M N} n^{M} n^{N} & =\kappa_{D}^{2} T_{M N} n^{M} n^{N}+\frac{\lambda}{2} H_{M N} n^{M} n^{N} \\
& =\kappa_{D}^{2} \frac{(\rho+p)}{a^{2}}+\lambda \frac{\ddot{a} \dot{a}^{2}}{a^{5}}(D-4)(D-3)(D-2)(D-1)-\lambda \frac{\dot{a}^{4}}{a^{6}}(D-4)(D-3)(D-2) \\
& =\kappa_{D}^{2} \frac{(\rho+p)}{a^{2}}+\lambda \frac{\dot{H}_{D} H_{D}^{2}}{a^{2}}(D-4)(D-3)(D-2)(D-1)+\lambda \frac{H_{D}^{4}}{a^{2}}(D-4)(D-3)(D-2)^{2},
\end{aligned}
$$

where we wrote $H_{D} \equiv \dot{a} / a$ to simplify our result.

\footnotetext{
${ }^{4}$ In the Appendix, we consider cosmological models in which spacetime is divided into a $d$-dimensional external spacetime and $m$ dimensional internal space, each of which with their own time-dependent scale factors.
} 
Clearly the first term in (50) is positive for matter that obeys the null energy condition, as usual. However, the second term can be negative if the time variation of the Hubble parameter $\dot{H}_{D}$ is large enough.

In particular, let us consider perturbative solutions for the metric 44 of the form

$$
a(t) \approx a_{0}(t)+\lambda a_{1}(t)+\ldots
$$

where $a_{0}(t)$ is the zeroth-order solution to the $\lambda=0$ Einstein equation

$$
\begin{aligned}
& \frac{\dot{a}_{0}^{2}}{a_{0}^{2}}=\frac{\kappa_{D}^{2}}{6} \rho \equiv\left(H_{D}^{(0)}\right)^{2} ; \\
& \frac{\ddot{a}_{0}}{a_{0}}=-\frac{\kappa_{D}^{2}}{12}(\rho+3 p) \equiv \dot{H}_{D}^{(0)}+\left(H_{D}^{(0)}\right)^{2},
\end{aligned}
$$

and $H_{D}^{(0)}$ is the corresponding zeroth-order Hubble parameter. Inserting $H_{D}^{(0)}$ into 50 , we obtain the expression

$$
R_{M N} n^{M} n^{N}=\kappa_{D}^{2} \frac{(\rho+p)}{a_{0}^{2}}-\lambda \kappa_{D}^{4} \frac{(D-4)(D-3)(D-2)}{24 a_{0}^{2}} \rho\left(\frac{D+1}{3} \rho+\frac{D-1}{p}\right)+\mathcal{O}\left(\lambda^{2}\right) .
$$

Assuming an equation of state $p=w \rho$, the second term is negative for equations of state $w>-\frac{D+1}{3(D-1)}$. The lower bound is always greater than $-1 / 2$, so most ordinary matter will satisfy this condition. In particular, a $D$-dimensional universe dominated by radiation $w=1 / 3$ can contribute a divergent term in the null Raychaudhuri equation.

Finally, consider a comoving, proper-time parameterized timelike geodesic described by the tangent vector

$$
u^{M}=(1,0,0,0) .
$$

The corrections to the timelike Raychaudhuri equation due to the Gauss-Bonnet corrections come from the last term

$$
\begin{aligned}
R_{M N} u^{M} u^{N} & =\kappa_{D}^{2}\left(T_{M N} u^{M} u^{N}+\frac{1}{2} T_{M}^{M}\right)+\frac{\lambda}{2} H_{M N} u^{M} u^{N} \\
& =\kappa_{D}^{2} \frac{(D-3) \rho+(D-1) p}{D-2}+\frac{\lambda}{2}(D-4)(D-3)(D-1)\left[2 \dot{H}_{D}+H_{D}^{2}\right] H_{D}^{2},
\end{aligned}
$$

where again we substituted $H_{D} \equiv \dot{a} / a$ to simplify our result. The first term is the typical term for matter, and is positive for matter that obeys the strong energy condition. The last term, proportional to $\lambda$, is the new contribution; we see that for $\dot{H}_{D} \sim-H_{D}^{2}$ (as is the case for any $a(t) \sim t^{n}$ time-dependence), this term can be negative, giving rise to a positive divergent term in the timelike Raychaudhuri equation, potentially opposing convergence.

We have seen in both the null and timelike Raychaudhuri equations the existence of terms that can give rise to a positive (divergent) contribution to the divergence. This does not necessarily mean that cosmology with EGB gravity can evade the singularity problem, just that the usual singularity theorems do not apply in a straightforward way to these backgrounds.

In particular, consider the EGB-corrected equations of motion for the metric (44) from (16). Examining the $(t t)$ component of 16 we have

$$
\frac{1}{2}(D-2)(D-1) \frac{\dot{a}^{2}}{a^{2}}=\kappa_{D}^{2} \rho-\frac{\lambda}{4}(D-4)(D-3)(D-2)(D-1) \frac{\dot{a}^{4}}{a^{4}} .
$$

For $\lambda \kappa_{D}^{2} \rho \ll 1$, solutions to 57 take the usual Einstein form; however, for $\lambda \kappa_{D}^{2} \rho \gg 1$, as we would expect to occur in the early universe near a big bang singularity, we have instead

$$
\frac{\dot{a}^{2}}{a^{2}} \sim \sqrt{\frac{\kappa_{D}^{2} \rho}{\lambda}} .
$$

It is clear that a curvature singularity still exists where $a(t) \rightarrow 0, R_{D} \rightarrow \infty$, despite the presence of the higher curvature terms. 


\section{BRANEWORLD GRAVITY}

We will consider a 5 -dimensional bulk spacetime (with 5-dimensional cosmological constant $\Lambda_{5}$ ) with coordinates $(t, \vec{x}, y)$ and metric $g_{M N}$ given by [25]

$$
d s^{2}=a(t, y)^{2} b(t, y)^{2}\left(-d t^{2}+d y^{2}\right)+a^{2}(t, y)\left(d r^{2}+r^{2} d \Omega^{2}\right),
$$

which is sufficiently general to capture the backreaction of the singular brane on the bulk as well as 4-dimensional homogeneous and isotropic cosmological evolution. The extra dimension has the range $y \in(-\infty, \infty)$; however, we will additionally impose a $\mathbb{Z}_{2}$ symmetry [26] $y \rightarrow-y$, so that the covering space is reduced to $y \in[0, \infty)$, as in the RS I scenario 27. We embed a (singular) 3 -brane at $y=0$ with unit normal $n^{M}=\left(0,0,0,0, \frac{1}{a b}\right)$ and induced metric $q_{\mu \nu}=g_{\mu \nu}-n_{\mu} n_{\nu}$; we will use capital Latin letters $M, N$ for 5 -dimensional coordinate indicies and lowercase Greek letters $\mu, \nu$ for 4 -dimensional brane indicies. The 5 -dimensional energy-momentum tensor has the form

$$
{ }^{(5)} T_{M N}=-\Lambda_{5} g_{M N}+\delta_{M}^{\mu} \delta_{N}^{\nu} S_{\mu \nu} \delta(y), \quad S_{\mu \nu}=-\sigma q_{\mu \nu}+\tau_{\mu \nu}
$$

where $\sigma$ is the brane tension and $\tau_{\mu \nu}$ is the brane matter energy momentum tensor. Note that the usual Minkowski RS I model has the solution

$$
d s^{2}=e^{-2 K(z)} \eta_{\mu \nu} d x^{\mu} d x^{\nu}+d z^{2} .
$$

This can be obtained from (59) under the limits and identifications

$$
\begin{aligned}
b(t, y) & \rightarrow 1 ; \\
a(t, y) & \rightarrow a(y)=e^{-K(z)} ; \\
z & =\int_{0}^{\infty} a(y) d y .
\end{aligned}
$$

Gravity on the 3-brane hypersurface is induced by its embedding in the extra dimensions; in particular, the induced 4-dimensional Einstein equations on the brane are 28]

$$
{ }^{(4)} R_{\mu \nu}-\frac{1}{2} q_{\mu \nu}{ }^{(4)} R=-\Lambda_{4} q_{\mu \nu}+\kappa_{4}^{2} \tau_{\mu \nu}+\kappa_{5}^{4} \pi_{\mu \nu}-E_{\mu \nu},
$$

where $\Lambda_{4}=\frac{1}{2} \kappa_{5}^{2}\left(\Lambda_{5}+\frac{1}{6} \kappa_{5}^{2} \sigma^{2}\right)$ and $\kappa_{4}^{2}=\frac{\kappa_{5}^{4} \sigma}{6}$, and the new tensors $\pi_{\mu \nu}, E_{\mu \nu}$ are defined as

$$
\begin{aligned}
\pi_{\mu \nu} & =\lim _{y \rightarrow 0}\left[-\frac{1}{4} \tau_{\mu \alpha} \tau_{\nu}^{\alpha}+\frac{1}{12} \tau \tau_{\mu \nu}+\frac{1}{8} q_{\mu \nu} \tau_{\alpha \beta} \tau^{\alpha \beta}-\frac{1}{24} q_{\mu \nu} \tau^{2}\right] \\
E_{\mu \nu} & =\lim _{y \rightarrow 0}\left[{ }^{(5)} C_{\mu \alpha \nu \beta} n^{\alpha} n^{\beta}\right]
\end{aligned}
$$

where ${ }^{(5)} C_{M N P Q}$ is the 5-dimensional Weyl tensor; see Appendix $\mathrm{C}$ for more details. The first two terms on the right-hand side of (63) are just the usual 4-dimensional cosmological constant and energy-momentum tensor sources, while the last two terms arise from the extra dimensional embedding of the brane. In order to put this into a form suitable for use with the Raychaudhuri equation we trace-reverse 63)

$$
\begin{aligned}
{ }^{(4)} R_{\mu \nu} & =\Lambda_{4} q_{\mu \nu}+8 \pi G_{N}\left(\tau_{\mu \nu}-\frac{1}{2} q_{\mu \nu} \tau_{\mu}^{\mu}\right)+\kappa_{5}^{4}\left(\pi_{\mu \nu}-\frac{1}{2} q_{\mu \nu} \pi_{\mu}^{\mu}\right)-\left(E_{\mu \nu}-\frac{1}{2} q_{\mu \nu} E_{\mu}^{\mu}\right) \\
& =\Lambda_{4} q_{\mu \nu}+8 \pi G_{N}\left(\tau_{\mu \nu}-\frac{1}{2} q_{\mu \nu} \tau_{\mu}^{\mu}\right)+H_{\mu \nu},
\end{aligned}
$$

where we rewrote the last two terms as a correction term $H_{\mu \nu}$ so that the corrections take the form (7) as outlined in the Introduction. The timelike and null 4-dimensional Raychaudhuri equations thus have the new terms arising from the braneworld

$$
\begin{aligned}
& \frac{d \theta}{d \tau}=-{ }^{(4)} R_{\mu \nu} u^{\mu} u^{\nu}+\ldots=-\kappa_{5}^{4}\left(\pi_{\mu \nu} u^{\mu} u^{\nu}+\frac{1}{2} \pi_{\mu}^{\mu}\right)+\left(E_{\mu \nu} u^{\mu} u^{\nu}+\frac{1}{2} E_{\mu}^{\mu}\right)+\ldots ; \\
& \frac{d \hat{\theta}}{d \lambda}=-{ }^{(4)} R_{\mu \nu} n^{\mu} n^{\nu}+\ldots=-\kappa_{5}^{4} \pi_{\mu \nu} n^{\mu} n^{\nu}+E_{\mu \nu} n^{\mu} n^{\nu}+\ldots
\end{aligned}
$$


where ... on the right-hand sides denote non-positive terms.

Assuming the brane energy-momentum tensor is of the perfect fluid form, with energy density $\rho(t)$ and pressure $p(t)$, we can immediately compute the $\pi_{\mu \nu}$ tensor contribution for the metric (59)

$$
\begin{array}{ll}
\pi_{t t}=\frac{1}{12} \rho^{2}(t) a^{2}(t, 0) b^{2}(t, 0) ; & \pi_{r r}=\frac{1}{12} \rho(t)(2 p(t)+\rho(t)) a^{2}(t, 0) ; \\
\pi_{\theta \theta}=\frac{1}{12} \rho(t)(2 p(t)+\rho(t)) a(t, 0)^{2} r^{2} ; & \pi_{\phi \phi}=\frac{1}{12} \rho(t)(2 p(t)+\rho(t)) ; a^{2}(t, 0) r^{2} \sin ^{2} \theta,
\end{array}
$$

where we evaluated the terms at $y=0$, while we compute $E_{\mu \nu}$ directly from the metric 59

$$
\begin{aligned}
& E_{t t}=\lim _{y \rightarrow 0} \frac{1}{2}\left[\frac{b^{\prime \prime}}{b}-\frac{b^{2}}{b^{2}}-\frac{\ddot{b}}{b}+\frac{\dot{b}^{2}}{b^{2}}\right] \equiv \lim _{y \rightarrow 0} \frac{1}{2} \beta(t, y) ; \\
& E_{r r}=\frac{1}{6} \lim _{y \rightarrow 0} \beta(t, y)=\frac{E_{\theta \theta}}{r^{2}}=\frac{E_{\phi \phi}}{r^{2} \sin ^{2} \theta},
\end{aligned}
$$

where a dot denotes a derivative with respect to $t$, and a prime denotes a derivative with respect to $y$. All of the components of the $E_{\mu \nu}$ tensor are proportional to the quantity $\beta(t, y)$, involving a second derivative $b^{\prime \prime}(t, y)$.

In order to evaluate the common quantity $\beta(t, y)$ appearing in $E_{\mu \nu}$ in the limit $y \rightarrow 0$, we need to use the 5dimensional Einstein equations ${ }^{(5)} R_{M N}-\frac{1}{2} g_{M N}^{(5)} R=\kappa_{5}^{2}{ }^{(5)} T_{M N}$ for the metric 59 and sources 60 , which take the form $[25]$

$$
\begin{aligned}
& (t t) \quad 3\left[2 \frac{\dot{a}^{2}}{a^{2}}+\frac{\dot{a} \dot{b}}{a b}-\frac{a^{\prime \prime}}{a}+\frac{a^{\prime} b^{\prime}}{a b}\right]=a^{2} b^{2} \kappa_{5}^{2}\left[\Lambda_{5}+(\rho+\sigma) \delta(y)\right] ; \\
& (y y) \quad 3\left[\frac{\ddot{a}}{a}-\frac{\dot{a} \dot{b}}{a b}-2 \frac{a^{\prime 2}}{a^{2}}-\frac{a^{\prime} b^{\prime}}{a b}\right]=a^{2} b^{2} \kappa_{5}^{2} \Lambda_{5} ; \\
& (t y) \quad 3\left[-\frac{\dot{a}^{\prime}}{a}+2 \frac{\dot{a} a^{\prime}}{a^{2}}+\frac{\dot{a} b^{\prime}}{a b}+\frac{a^{\prime} \dot{b}}{a b}\right]=0 ; \\
& (r r) \&(i j) \quad\left[3 \frac{\ddot{a}}{a}+\frac{\ddot{b}}{b}-\frac{\dot{b}^{2}}{b^{2}}-3 \frac{a^{\prime \prime}}{a}-\frac{b^{\prime \prime}}{b}+\frac{b^{\prime 2}}{b^{2}}\right]=a^{2} b^{2} \kappa_{5}^{2}\left[\Lambda_{5}+(\sigma-p) \delta(y)\right] .
\end{aligned}
$$

Integrating the $(t t)$ and $(i j)$ equations across the brane gives rise to

$$
\left.\frac{a^{\prime}}{a}\right|_{y=0}=\frac{1}{6} a(t, 0) b(t, 0) \kappa_{5}^{2}(\rho+\sigma),\left.\quad \frac{b^{\prime}}{b}\right|_{y=0}=-\frac{1}{2} a(t, 0) b(t, 0) \kappa_{5}^{2}(\rho+p) .
$$

In addition, the restriction of the $(t y)$ component of the Einstein equations to the brane at $y=0$ takes the form of the usual energy-momentum conservation equation on the brane

$$
\dot{\rho}+\left.3 \frac{\dot{a}}{a}\right|_{y=0}(\rho+p)=0 .
$$

Finally, restricting the $(y y)$ component of the Einstein equations to the brane at $y=0$ we can obtain a "Hubble"-like equation

$$
\left(\frac{1}{a b} \frac{\dot{a}}{a}\right)^{2}=\kappa_{5}^{4} \frac{(\rho+\sigma)^{2}}{36}+\kappa_{5}^{2} \frac{\Lambda_{5}}{6}+\frac{\mu}{a^{4}}
$$

Where the last term arises as an integration constant and is known as the "dark radiation" term [29 31]. We will assume $\mu \geq 0$.

We can rearrange 76 to write

$$
\beta(t, y)=\frac{b^{\prime \prime}}{b}-\frac{b^{\prime 2}}{b^{2}}-\frac{\ddot{b}}{b}+\frac{\dot{b}^{2}}{b^{2}}=3\left(\frac{\ddot{a}}{a}-\frac{a^{\prime \prime}}{a}\right)-a^{2} b^{2} \kappa_{5}^{2}\left(\Lambda_{5}-p \delta(y)\right) .
$$

Subtracting (74) and (73), this then becomes

$$
\beta(t, y)=6\left(\frac{\dot{a}^{2}}{a^{2}}-\frac{a^{\prime 2}}{a^{2}}\right)-a^{2} b^{2} \kappa_{5}^{2}\left(\Lambda_{5}+(\rho+p) \delta(y)\right) .
$$


Using the jump condition (77) and the Hubble equation $(79)$, this simplifies considerably to

$$
\beta(t, y)=6 \mu \frac{b^{2}}{a^{2}}-a^{2} b^{2} \kappa_{5}^{2}(\rho+p) \delta(y)
$$

The $E_{\mu \nu}$ tensor is evaluated in the limit $y \rightarrow 0$; since the delta-function does not contribute in this limit, we have

$$
E_{\mu \nu} \propto \lim _{y \rightarrow 0} \beta(t, y)=6 \mu \frac{b^{2}}{a^{2}},
$$

thus the Weyl tensor term $E_{\mu \nu}$ only potentially contributes to the induced Einstein equations on the brane through the dark radiation term. However, the induced Einstein equations (63) are not closed, and must be supplemented by additional constraints [28. Examining these constraints for our background and matter content in Appendix C, we find that the dark radiation term $\mu$ must vanish, so that the $E_{\mu \nu}$ contributions vanish as well.

In order to evaluate the correction terms 6869 , we will take the proper-time parameterized timelike vector and affine null vectors on the brane to be

$$
\begin{aligned}
u^{\mu} & =\left(\frac{1}{a(t, 0) b(t, 0)}, 0,0,0\right), \\
n^{\mu} & =\left(\frac{1}{a(t, 0) b(t, 0)}, \pm \frac{1}{a(t, 0)}, 0,0\right),
\end{aligned}
$$

where the \pm refers to radially outgoing/ingoing null rays. We can then compute the additional braneworld terms

$$
\begin{aligned}
& H_{\mu \nu} u^{\mu} u^{\nu}=\kappa_{5}^{4} \rho\left(\rho+\frac{3}{2} p\right) ; \\
& H_{\mu \nu} n^{\mu} n^{\nu}=\kappa_{5}^{4} \frac{\rho^{2}}{12} .
\end{aligned}
$$

We see that the braneworld terms only contribute positively to the additional terms for null vectors, while the additional contributions for timelike vectors can be negative if $p<-2 / 3 \rho$. Including these results, we have the following expressions for the timelike and null Raychaudhuri equations on the brane

$$
\begin{aligned}
& \frac{d \theta}{d \tau}=\Lambda_{4}-\frac{1}{2} G_{N}(\rho+3 p)-\kappa_{5}^{4} \rho\left[\rho+\frac{3}{2} p\right]+\ldots ; \\
& \frac{d \hat{\theta}}{d \lambda}=-G_{N}(\rho+p)-\kappa_{5}^{4} \frac{\rho^{2}}{12}+\ldots,
\end{aligned}
$$

where again ... refer to strictly non-positive terms. Thus, we see that the corrections to the induced Einstein equations on the brane due to the braneworld embedding of the brane only contribute to convergence for the null Raychaudhuri equation. However, the additional contributions do add divergent (positive) terms to the right-hand side of the timelike Raychaudhuri equation; we note, though, that the second term in Eq.(88), which is present in un-modified 4-dimensional General Relativity, is also divergent in the presence of matter that violates the strong energy condition, $p<-1 / 3 \rho$.

Is the presence of the additional positive term in Eq. 88 arising from induced gravity on the brane sufficient to avoid a singularity? Rewriting the Friedmann-like equation Eq. 79 using the relations $\Lambda_{4}=\frac{1}{2} \kappa_{5}^{2}\left(\Lambda_{5}+\frac{1}{6} \kappa_{5}^{2} \sigma^{2}\right)$ and $\kappa_{4}^{2}=\frac{\kappa_{5}^{4} \sigma}{6}$ and by reparameterizing the time variable on the brane into cosmic time, $d t^{\prime}=a(t, 0) b(t, 0) d t$, we have [32, 33]

$$
\left(\frac{d a / d t^{\prime}}{a}\right)^{2}=\frac{\kappa_{4}^{2}}{3} \rho\left(1+\frac{\rho}{2 \sigma}\right)+\frac{\Lambda_{4}}{3} .
$$

Notice that at late times, when the energy density is much smaller than the brane tension $\rho \ll \sigma$, we recover the usual Friedmann equation of General Relativity. However, at early times $\rho \geq \sigma$, assuming an equation of state $\rho=w p$ $(w \neq 1)$ implies $\rho \propto a^{-3(1+w)}$ (recall that local energy conservation on the brane Eq. 78 is unchanged) so that [34, 35]:

$$
\frac{d a / d t^{\prime}}{a} \sim \frac{a^{-3(1+w)}}{\sqrt{\sigma}} ; \quad \Rightarrow \quad a \sim\left((1+w) \frac{t^{\prime}}{\sqrt{\sigma}}\right)^{\frac{1}{3(1+w)}},
$$

which diverges as $t^{\prime} \longrightarrow 0$. Thus, we expect the Big Bang singularity persists in braneworld induced gravity models as well. 


\section{F(R) THEORIES}

Higher curvature gravity theories, collectively known as $f(R)$ theories, have been studied both for its improved short distance properties, although at the expense of introducing ghosts [36], as well as for their potential for avoiding singularities, and as a model of inflation and an alternative model for dark energy. We start with the action for $f(R)$ gravity in the so-called Jordan frame $37+40$.

$$
S=\frac{1}{\kappa_{4}^{2}} \int d^{4} x \sqrt{-g} f(R)+S\left(g_{\mu \nu}, \psi\right),
$$

where the last term represents matter action.

From this action, variation of the metric gives rise to the modified Einstein equations [38.

$$
R_{\mu \nu} f^{\prime}(R)=\kappa_{4}^{2} T_{\mu \nu}+\frac{1}{2} g_{\mu \nu} f(R)+\left(\nabla_{\mu} \nabla_{\nu}-g_{\mu \nu} \square\right) f^{\prime}(R) .
$$

From this we can write the null and timelike Raychaudhuri equations for $f(R)$ gravity:

$$
\begin{aligned}
& \frac{d \hat{\theta}}{d \lambda}=-\frac{\hat{\theta}^{2}}{2}-\left[\frac{\kappa_{4}^{2}}{f^{\prime}(R)} T_{\mu \nu}+\frac{1}{2} g_{\mu \nu} \frac{f(R)}{f^{\prime}(R)}+\frac{1}{f^{\prime}(R)}\left(\nabla_{\mu} \nabla_{\nu}-g_{\mu \nu} \square\right) f^{\prime}(R)\right] n^{\mu} n^{\nu} \\
& \frac{d \theta}{d \tau}=-\frac{\theta^{2}}{3}-\left[\frac{\kappa_{4}^{2}}{f^{\prime}(R)} T_{\mu \nu}+\frac{1}{2} g_{\mu \nu} \frac{f(R)}{f^{\prime}(R)}+\frac{1}{f^{\prime}(R)}\left(\nabla_{\mu} \nabla_{\nu}-g_{\mu \nu} \square\right) f^{\prime}(R)\right] u^{\mu} u^{\nu}
\end{aligned}
$$

in terms of the expansions $\hat{\theta}, \theta$ for null and timelike geodesics $n^{\mu}(\lambda), u^{\mu}(\tau)$ parameterized by affine and proper times $\lambda, \tau$, respectively.

Since we are interested in singularities with larger curvatures, we will consider an $f(R)$ theory consisting of the usual scalar curvature plus a perturbative correction of higher power in the curvature $f(R)=R\left[1+\left(\ell_{P l}^{2} R\right)^{n-1}\right]$, where we will take the Planck length $\ell_{P l}$ to control the strength of the corrections, and $n$ is a positive integer. Since we are interested in the large curvature regime, i.e. $R \gg 1 / \ell_{P l}^{2}$, we will consider the case where the second term in $f(R)$ dominates so that $f \sim R^{n}$. In this case, the equations of motion are dominated by the higher curvature correction terms, which act as an effective energy-momentum tensor.

Substituting this into the Raychaudhuri equations 9495, we obtain

$$
\begin{aligned}
& \frac{d \hat{\theta}}{d \lambda}=-\frac{\hat{\theta}^{2}}{2}-\left[\frac{\kappa_{4}^{2} T_{\mu \nu}}{2 n \ell_{P l}^{2 n-2} R^{n-1}}+\frac{R}{2 n} g_{\mu \nu}+\frac{1}{n R^{n-1}}\left(\nabla_{\mu} \nabla_{\nu}-g_{\mu \nu} \square\right) R^{n-1}\right] n^{\mu} n^{\nu} . \\
& \frac{d \theta}{d \tau}=-\frac{\theta^{2}}{3}-\left[\frac{\kappa_{4}^{2} T_{\mu \nu}}{2 n \ell_{P l}^{2-2} R^{n-1}}+\frac{R}{2 n} g_{\mu \nu}+\frac{1}{n R^{n-1}}\left(\nabla_{\mu} \nabla_{\nu}-g_{\mu \nu} \square\right) R^{n-1}\right] u^{\mu} u^{\nu} .
\end{aligned}
$$

Note that (i) the sign of the second term in square brackets in each equation is opposite to the $T_{\mu \nu}$ term, and (ii) the sign of the last term in each equation depends on the spacetime under consideration.

Next, we consider homogeneous and isotropic cosmological backgrounds with the metric

$$
d s^{2}=-d t^{2}+a(t)^{2}\left[\frac{d r^{2}}{1-k r^{2}}+r^{2}\left(d \theta^{2}+\sin ^{2} \theta d \phi^{2}\right)\right]
$$

and corresponding scalar curvature

$$
R=6\left(\frac{\ddot{a}}{a}+\frac{\dot{a}^{2}}{a^{2}}+\frac{k}{a^{2}}\right) .
$$

The null Raychaudhuri equation (96, using $n^{\mu} n^{\nu} g_{\mu \nu}=0$, becomes

$$
\frac{d \hat{\theta}}{d \lambda}=-\frac{\hat{\theta}^{2}}{2}-\frac{\kappa_{4}^{2} T_{\mu \nu} n^{\mu} n^{\nu}}{2 n \ell_{P l}^{2 n-2} R^{n-1}}+\frac{1}{n R^{n-1}}\left(n^{t}\right)^{2} \partial_{t}^{2} R^{n-1},
$$

while the timelike Raychaudhuri equation (97) becomes

$$
\frac{d \theta}{d \tau}=-\frac{\theta^{2}}{3}-\left[\frac{\kappa_{4}^{2} T_{\mu \nu}}{2 n \ell_{P l}^{2 n-2} R^{n-1}}+\frac{3}{n}\left(\frac{\ddot{a}}{a}+\frac{\dot{a}^{2}}{a^{2}}+\frac{k}{a^{2}}\right) g_{\mu \nu}\right] u^{\mu} u^{\nu} .
$$


When the cosmological evolution is dominated by the curvature corrections, we have an effective perfect fluid

$$
p_{\text {eff }}=w_{\text {eff }} \rho_{\text {eff }}, \quad w_{\text {eff }}=-\frac{6 n^{2}-7 n-1}{6 n^{2}-9 n+3},
$$

with scale factor $\left(w_{\text {eff }} \neq 1\right)$ :

$$
a=a_{0} t^{\frac{2}{3\left(1+w_{\mathrm{eff}}\right)}}, \frac{\dot{a}}{a}=\frac{2}{3\left(1+w_{\mathrm{eff}}\right) t}, \frac{\ddot{a}}{a}=-\frac{2\left(1+3 w_{\mathrm{eff}}\right)}{9\left(1+w_{\mathrm{eff}}\right)^{2} t^{2}}, \frac{\ddot{a}}{a}+\frac{\dot{a}^{2}}{a^{2}}+\frac{k}{a^{2}}=\frac{2\left(1-3 w_{\mathrm{eff}}\right)}{9\left(1+w_{\mathrm{eff}}\right)^{2} t^{2}}+\frac{k}{a_{0}^{2} t^{4 / 3\left(1+w_{\mathrm{eff}}\right)}} .
$$

We can substitute this solution for the scale factor into the Raychaudhuri equations to obtain

$$
\begin{aligned}
& \frac{d \hat{\theta}}{d \lambda}=-\frac{\hat{\theta}^{2}}{2}+\frac{1}{n\left\{\frac{2\left(1-3 w_{\text {eff }}\right)}{3 n\left(1+w_{\text {eff }}\right)^{2} t^{2}}+\frac{3 k}{n a_{0}^{2} t^{4 / 3\left(1+w_{\text {eff }}\right)}}\right\}^{n-1}} \times \\
& {\left[-\frac{\kappa_{4}^{2} T_{\mu \nu} n^{\mu} n^{\nu}}{2 \ell_{P l}^{2 n-2}}+\left(n^{t}\right)^{2} \partial_{t}^{2}\left\{\frac{2\left(1-3 w_{\text {eff }}\right)}{3 n\left(1+w_{\text {eff }}\right)^{2} t^{2}}+\frac{3 k}{n a_{0}^{2} t^{4 / 3\left(1+w_{\text {eff }}\right)}}\right\}^{n-1}\right] \text {; }}
\end{aligned}
$$

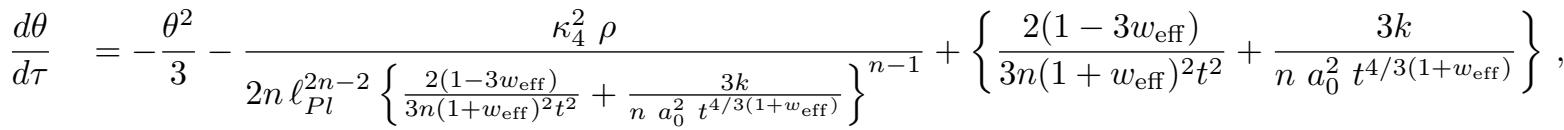

where we considered the rest frame of the fluid streamlines, for which $T_{\mu \nu} u^{\mu} u^{\nu}=\rho$, and used $u^{2}=-1$.

Since we are primarily interested in the corrected Raychaudhuri equation close to the putative Big-Bang singularity, i.e. pre-inflation with $w \neq-1$, we ignore the energy-momentum term in Eqs. (104 105), since this term becomes sub dominant at larger curvatures. Furthermore, omitting exotic matter from our discussions, i.e. $w \nless \leq-1 / 3$ such that the $k$ term can also be ignored, we arrive at the rather simple form for the Raychaudhuri equations

$$
\begin{aligned}
& \frac{d \hat{\theta}}{d \lambda}=-\frac{\hat{\theta}^{2}}{2}+\frac{2(n-1)(2 n-1)\left(n^{t}\right)^{2}}{n t^{2}} \\
& \frac{d \theta}{d \tau}=-\frac{\theta^{2}}{3}+\frac{2\left(1-3 w_{\text {eff }}\right)}{3 n\left(1+w_{\text {eff }}\right)^{2} t^{2}}=-\frac{\theta^{2}}{3}+\frac{3\left(4 n^{2}-5\right)\left(2 n^{2}-3 n+1\right)}{(n-2)^{2} t^{2}}
\end{aligned}
$$

As can be seen, the second term in both Eqs. 106 107) are repulsive and dominate over the first term (due to the factor of $1 / t^{2}$ therein) for all $n$. This causes geodesics to not to converge in this epoch, potentially preventing the formation of a singularity. At later epochs, e.g. during inflation and thereafter, that term is sub-dominant as are additional terms originating in an actual perfect fluid described by the equation $p=w \rho$, with $-1 \leq w \leq 1 / 3$. Therefore the standard conclusions from the Raychaudhuri equation and the singularity theorems hold. Note that while in [38], the authors consider constant curvature cosmological metrics (Einstein spaces), in 39 the authors consider theories of the form $f(R)=R+\alpha / R^{n}$ with $\alpha<0$ and $n \in \mathbb{R}$. These are relevant to late time acceleration, as an alternative to dark energy. As mentioned above, here we are primarily concerned with very early times, to examine effects of repulsive terms (if any) near the initial singularity. Furthermore, similar to the above references we too assume that the standard energy conditions are valid for the cosmological fluids.

\section{LOOP QUANTUM COSMOLOGY}

In loop quantum cosmology, one starts with the standard large scale homogeneous and isotropic model of the Universe, described by metric (98), and obtains an effective Hamiltonian, incorporating the discrete quantum nature of spacetime at the fundamental level, quantum back-reaction and the behavior of the scale factor at very small length scales 41,43

$$
\mathcal{H}_{\text {eff }}=-\frac{6}{\kappa_{4}^{2} \gamma^{2}} \frac{\sin ^{2}(\lambda \beta)}{\lambda^{2}} V+\mathcal{H}_{\text {matter }}
$$

where $\lambda$ is a measure of fundamental discreteness, $\gamma$ the Immirizi parameter, $V$ the volume, $\beta(=\gamma H)$ its conjugate, and $\mathcal{H}_{\text {matter }}$ the matter Lagrangian. Next, using the Hamiltonian constraint $\mathcal{H}_{\text {eff }} \approx 0$ and the Hamilton's equation for $V$, namely $\dot{V}=\left\{V, \mathcal{H}_{e f f}\right\}$, one obtains the 'Loop Quantum Corrected Raychaudhuri Equation'

$$
\frac{\ddot{a}}{a}=\dot{H}+H^{2}=-\frac{\kappa_{4}^{2}}{4}\left[\rho\left(1-\frac{\rho}{\rho_{c}}\right)+3\left\{P\left(1-\frac{2 \rho}{\rho_{c}}\right)-\frac{\rho^{2}}{\rho_{c}}\right\}\right]
$$


where: $\rho_{c} \equiv \frac{3}{8 \pi \gamma^{2} \lambda^{2} V^{2}}$.

By substituting [44, 45]

$$
\begin{aligned}
& \frac{\dot{a}}{a}=\frac{\theta}{3}, \\
& \hat{\theta}=\frac{2}{a^{2}}\left[\dot{a}-\frac{1}{r}\right]
\end{aligned}
$$

for timelike and null geodesics, respectively, we can obtain the corresponding Raychaudhuri equations

$$
\begin{aligned}
\frac{d \theta}{d \tau} & =-\frac{\theta^{2}}{3}-\frac{\kappa_{4}^{2}}{4}\left[\rho\left(1-\frac{\rho}{\rho_{c}}\right)+3\left\{P\left(1-\frac{2 \rho}{\rho_{c}}\right)-\frac{\rho^{2}}{\rho_{c}}\right\}\right] \\
\frac{d \hat{\theta}}{d \lambda} & =-\frac{\hat{\theta}^{2}}{2}+\frac{2}{a^{2}} \dot{H} \\
& =\hat{\theta}^{2}-\frac{2}{\left(a^{2} r\right)^{2}}-\frac{2 \theta}{a^{2} r}-\frac{\kappa_{4}^{2}}{2 a^{2}}\left[\rho\left(1-\frac{\rho}{\rho_{c}}\right)+3\left\{P\left(1-\frac{2 \rho}{\rho_{c}}\right)-\frac{\rho^{2}}{\rho_{c}}\right\}\right]
\end{aligned}
$$

Note that there are three positive (repulsive) terms in the RHS of the RE (112), (113), effective near $\rho \simeq \rho_{c}$, i.e. in the early Universe. Since the above equations were derived using a non-perturbative quantization scheme, without assuming a fixed background metric and allowing for back-reaction, one expects that they will hold good all the way to $t \rightarrow 0$. At this point, the repulsive terms take over (since $\rho \rightarrow \infty)$ and the singularity theorems and their conclusions cease to hold.

\section{CONCLUSIONS}

In this paper, we have examined the effects of several different alternative theories of gravity on the geodesic convergence properties of the Raychaudhuri equation in some simple backgrounds, and found some cases where these corrections provide repulsive terms.

In the case of string theory, we studied the leading order corrections to the Einstein Equations (for constant dilaton and vanishing form fields) arising in the form of Einstein-Gauss-Bonnet gravity. We did not find repulsive contributions to the Raychaudhuri equation for $D$-dimensional black hole backgrounds, which is consistent with known exact black hole solutions in Einstein-Gauss-Bonnet gravity, which still contain singularities. We did find repulsive terms for $D$-dimensional cosmological backgrounds, however these terms do not apear to be significant enough to prevent the existence of a Big Bang singularity, at least at leading order. It is therefore interesting that the string theory corrections to the Raychaudhuri equation do not have a definite sign, but instead the sign of the corrections depends on the background under consideration. We note that our results here should be taken as preliminary steps towards analyzing the potential for string theory corrections to resolve singularities. In particular, we have only examined the leading-order pure gravity corrections, setting the dilaton to a constant and form fields to zero. Allowing these fields to be dynamical will give rise to additional terms in the Raychaudhuri equation that could act repulsively. Additionally, the leading corrections we considered here vanish for type IIA/IIB string theory; it would be interesting, though technically challenging, to include the next order of corrections.

We also considered corrections to the Einstein equations coming from induced gravity on a brane embedded in a warped 5-dimensional bulk. In this case, the corrections show up as quadratic in the brane-localized energymomentum tensor. For a cosmological background we found that the corrections to the null Raychaudhuri equation always increase convergence, while the corrections ot the timelike Raychaudhuri equation can give rise to repulsive terms when the brane matter is described by a perfect fluid with pressure $p<-2 / 3 \rho$. Nonetheless, it appears that a Big Bang singularity persists in this case as well. It would be interesting to study these corrections for black hole backgrounds, as well as explore the role that bulk fields play in providing additional constraints.

We examined corrections for curvature-dominated $f(R)$ theories of the form $f(R)=R\left[1+\left(\ell_{P l} R\right)^{n-1}\right]$, where $\ell_{P l}$ is the Planck length and $n$ is a positive integer. We found that the $f(R)$ corrections can contribute repulsive terms to the timelike and null Raychaudhuri equations for $w_{\text {eff }}>-1 / 3$, where $w_{\text {eff }}(n)=-\left(6 n^{2}-7 n-1\right) /\left(6 n^{2}-9 n+3\right)$ is the effective equation of state of the curvature corrections. It would be interesting to study these corrections further to examine whether they do indeed lead to a resolution of the cosmological singularity.

Finally, we also considered corrections to the Friedmann equation for Loop Quantum Cosmology. These corrections give rise to repulsive terms in both the timelike and null Raychaudhuri equations, suggesting a resolution of the Big Bang singularity. Note that in this case the results are non-perturbative in nature. It is important to explore $f(R)$ theories and Loop Quantum Cosmology further to see if indeed all possible geodesics are complete in these theories, 
and if so, whether there exists another criterion for the existence of singularities. If not, the corresponding spacetimes should play an important role near the normally singular regions inside black holes and in cosmology.

In all of our examples we have considered simple isotropic and/or homogeneous backgrounds, and it would be interesting to study how robust our results are to anisotropic or inhomogeneous deviations. Since the power of the singularity theorems is in their general applicability, and generic situations likely would not possess such a high degree of symmetry, it is not clear whether the additional terms to the Raychaudhuri equation would continue to be repulsive in more general backgrounds with less symmetry. We leave this general analysis for future work. In addition, all of our analysis has focused on the corrections to the classical equations of motion from alternative theories of gravity. It would be interesting to examine quantum effects (which depend on the wavefunction of the fluid) over and above the above classical terms. However, since these effects are always repulsive in nature, and prevents the quantal trajectories (quantum counterparts of geodesics) from crossing, such effects should reinforce the above conclusion [46, 47.

\section{Acknowledgment}

This work is supported by the Natural Sciences and Engineering Research Council of Canada. SSH would like to thank Perimeter Institute, where part of the work was done. DJB is supported by a PHD fellowship from the South African National Institute for Theoretical Physics. NM is supported by the South African Research Chairs Initiative of the Department of Science and Technology and the National Research Foundation of South Africa. Any opinion, finding and conclusion or recommendation expressed in this material is that of the authors and the NRF does not accept any liability in this regard.

\section{Appendix A: Raychaudhuri Equations in $D$ Dimensions}

\section{Timelike}

Consider a congruence of timelike geodesics parameterized by proper time $\tau$ with tangent vectors $u^{M}(\tau)$ satisfying $u^{M} u_{M}=-1$ and $u^{M} \nabla_{M} u^{N}=0$ for some $D$-dimensional metric $g_{M N}$. We can define the "spatial" (or transverse) metric as

$$
h_{M N} \equiv g_{M N}+u_{M} u_{N},
$$

which is transverse to the tangent vectors $u^{M} h_{M N}=0$. We will define the following quantities:

$$
\begin{aligned}
\theta \equiv \nabla_{M} u^{M} & \text { Expansion scalar } \\
\sigma_{M N}=\frac{1}{2}\left(\nabla_{M} u_{N}+\nabla_{N} u_{M}\right)-\frac{1}{D-1} h_{M N} \theta & \text { Shear tensor } \\
\omega_{M N}=\frac{1}{2}\left(\nabla_{M} u_{N}-\nabla_{N} u_{M}\right) & \text { Twist tensor }
\end{aligned}
$$

Note that $\sigma_{M N}, \omega_{M N}$ are purely spatial (or transverse), since $u^{M} \sigma_{M N}=0=u^{M} \omega_{M N}$. In addition, $\omega_{M N}=0$ for tangent vectors that are hypersurface orthogonal, as will be the case for all of the tangent vectors considered in this paper. Following [48, the expansion obeys the Raychaudhuri equation

$$
\frac{d \theta}{d \tau}=-\frac{\theta^{2}}{D-1}-\sigma_{M N} \sigma^{M N}+\omega_{M N} \omega^{M N}-R_{M N} u^{M} u^{N} .
$$

Since $\sigma_{M N}$ is purely spatial, the second term is non-positive, and as mentioned above we will be considering cases where $\omega_{M N}=0$, so that the right-hand side of Eq. A5 is non-positive as long as $R_{M N} u^{M} u^{N} \geq 0$.

\section{Null}

The derivation of the null Raychaudhuri equation proceeds in a similar way as the timelike case above, with an additional complication in identifying the transverse directions to the null geodesic.

Consider a congruence of null geodesics parameterized by affine parameter $\lambda$ with tangent vectors $n^{M}(\lambda)$, satisfying $n^{M} n_{M}=0$ and $n^{M} \nabla_{M} n^{N}=0$. In order to define the transverse directions to the geodesic we need an "auxiliary" 
null vector $k^{M}$ such that ${ }^{5} k^{M} k_{M}=0$ and $n^{M} k_{M}=-1$. We define the transverse metric to the null geodesics

$$
\hat{h}_{M N}=g_{M N}+n_{M} k_{N}+k_{M} n_{M}
$$

which has dimension $g^{M N} \hat{h}_{M N}=D-2$ and is transverse to both $n^{M}$ and $k^{M}: n^{M} \hat{h}_{M N}=0=k^{M} \hat{h}_{M N}$. We then proceed as in the timelike case by defining:

$$
\begin{aligned}
\hat{\theta} \equiv \nabla_{M} n^{M} & \text { Expansion scalar } \\
\hat{\sigma}_{M N} \equiv \frac{1}{2}\left(\nabla_{M} n_{N}+\nabla_{N} n_{M}\right)-\frac{1}{D-2} \hat{h}_{M N} \hat{\theta} & \text { Shear tensor } \\
\hat{\omega}_{M N} \equiv \frac{1}{2}\left(\nabla_{M} n_{N}-\nabla_{N} n_{M}\right) & \text { Twist tensor }
\end{aligned}
$$

As before, $\sigma_{M N}, \omega_{M N}$ are transverse to the null geodesics. The expansion obeys the Raychaudhuri equation

$$
\frac{d \hat{\theta}}{d \lambda}=-\frac{\hat{\theta}^{2}}{D-2}-\hat{\sigma}_{M N} \hat{\sigma}^{M N}+\hat{\omega}_{M N} \hat{\omega}^{M N}-R_{M N} n^{M} n^{N} .
$$

As in the timelike case, $\omega_{M N}=0$ for geodesics that are hypersurface orthogonal; further, since $\sigma_{M N}$ is purely spatial the second term in Eq.A10 is non-positive, so that the entire right-hand side is non-positive as well as long as $R_{M N} n^{M} n^{N} \geq 0$

\section{Appendix B: Gauss-Bonnet Corrections in Anisotropic Cosmology}

We will examine the correction terms $H_{M N}$ for a $D$-dimensional background consisting of a $d$-dimensional flat, homogeneous spacetime, with coordinates $\left\{r, \theta^{i}\right\}$, and a $m$-dimensional homogeneous spacetime, with coordinates $\left\{y^{m}\right\}$, each with their own scale factor

$$
d s^{2}=-d t^{2}+a(t)^{2}\left(d r^{2}+r^{2} \hat{d}_{d-1}^{2}\right)+b(t)^{2} \tilde{g}_{m n} d y^{m} d y^{n},
$$

where $D=d+m+1$.

The Gauss-Bonnet scalar is

$$
\begin{aligned}
& R_{G B}^{2}=R_{A B C D} R^{A B C D}-4 R_{M N} R^{M N}+\left(R_{D}\right)^{2} \\
& =4 d(d-1)(d-2) \frac{\ddot{a}}{a} \frac{\dot{a}^{2}}{a^{2}}+d(d-1)(d-2)(d-3) \frac{\dot{a}^{4}}{a^{4}} \\
& +4 m(m-1)(m-2) \frac{\ddot{b}}{b} \frac{\dot{b}^{2}}{b^{2}}+m(m-1)(m-2)(m-3) \frac{\dot{b}^{4}}{b^{4}}
\end{aligned}
$$

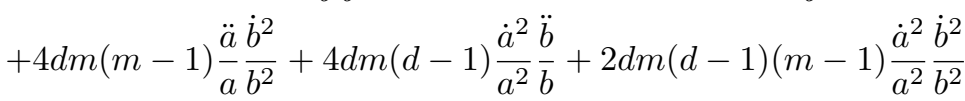

$$
\begin{aligned}
& +\frac{\tilde{R}_{m}}{b^{2}}\left[4 d \frac{\ddot{a}}{a}+2 d(d-1) \frac{\dot{a}^{2}}{a^{2}}+4(m-2) \frac{\ddot{b}}{b^{3}}-6 m(m-1) \frac{\dot{b}^{2}}{b^{2}}-3 \frac{\tilde{R}_{m}}{b^{2}}\right] \text {. }
\end{aligned}
$$

Note that $R_{G B}^{2}$ simplifies considerably when $d=3$ and $m=1,2$, corresponding to $D=5$ or 6 -dimensional spacetime, respectively, and the internal space is flat $\tilde{R}_{m}=0$

$$
\left.R_{G B}^{2}\right|_{d=3, m=1,2}=24 \frac{\ddot{a} \dot{a}^{2}}{a^{3}}+24 m \frac{\dot{a}^{2}}{a^{2}} \frac{\ddot{b}}{b},
$$

though this doesn't seem to give any particularly useful interpretation.

\footnotetext{
${ }^{5} k^{M}$ need not be a geodesic, and indeed in most cases it is not possible for both $k^{M}$ and $n^{M}$ to be geodesics and cross-normalized to a constant.
} 
We can now compute the correction terms

$$
\begin{aligned}
H_{t t}= & \frac{g_{t t}}{2} R_{G B}^{2}-2 R_{D} R_{t t}+4 R_{t t} g^{t t} R_{t t}+4\left(g^{r r}\right)^{2} R_{r r} R_{t r t r}+4 g^{i i^{\prime}} g^{j j^{\prime}} R_{i^{\prime} j^{\prime}} R_{t i t j}+4 R^{m n} R_{t m t n}-2 R_{t A B C} R_{t}^{A B C} \\
= & -\frac{1}{2} d(d-1)(d-2)(d-3) \frac{\dot{a}^{4}}{a^{4}}-\frac{1}{2} m(m-1)(m-2)(m-3) \frac{\dot{b}^{4}}{b^{4}}-d m(d-1)(m-1) \frac{\dot{a}^{2}}{a^{2}} \frac{\dot{b}^{2}}{b^{2}} \\
& +\frac{\tilde{R}_{m}}{b^{2}}\left[-d(d-1) \frac{\dot{a}^{2}}{a^{2}}+3 m(m-1) \frac{\dot{b}^{2}}{b^{2}}+\frac{3}{2} \frac{\tilde{R}_{m}}{b^{2}}\right] ; \\
H_{r r}= & \frac{g_{r r}}{2} R_{G B}^{2}-2 R_{D} R_{r r}+4 R_{r r} g^{r r} R_{r r}+4 R_{t t}\left(g^{t t}\right)^{2} R_{r t r t}+4 R_{i^{\prime} j^{\prime}} g^{i i^{\prime}} g^{j j^{\prime}} R_{r i r j}-2 R_{r A B C} R_{r}^{A B C} \\
= & 2(d-1)(d-2)(d-3) \frac{\ddot{a} \dot{a}^{2}}{a}+\frac{1}{2}(d-1)(d-2)(d-3)(d-4) \frac{\dot{a}^{4}}{a^{2}} \\
& +2 m(m-1)(m-2) a^{2} \frac{\ddot{b}^{2}}{b^{3}}+m(m-1)(m-2)(m-3) a^{2} \frac{\dot{b}^{4}}{b^{4}} \\
& +2 m(m-1)(d-1) a \ddot{a} \frac{\dot{b}^{2}}{b^{2}}+2 m(d-1)(d-2) \dot{a}^{2} \frac{\ddot{b}}{b}+m(m-1)(d-1)(d-2) \dot{a}^{2} \frac{\dot{b}^{2}}{b^{2}} \\
& +a^{2} \frac{\tilde{R}_{m}}{b^{2}}\left[2 d \frac{\ddot{a}}{a}+d(d-1) \frac{\dot{a}^{2}}{a^{2}}+2(m-2) \frac{\ddot{b}}{b^{3}}-3 m(m-1) \frac{\dot{b}^{2}}{b^{2}}-\frac{3}{2} \frac{\tilde{R}_{m}}{b^{2}}\right] .
\end{aligned}
$$

Notice that $H_{t t}$ vanishes identically for all $d=3, m=1,2$ and a flat internal space $\tilde{R}_{m}=0$, while $H_{r r}$ is considerably more complex.

\section{a. Raychaudhuri Corrections}

We now consider a $d$-dimensional radial, null, affine tangent vector

$$
n^{M}=\left(\frac{1}{a(t)}, \pm \frac{1}{a(t)^{2}}, \overrightarrow{0}, \overrightarrow{0}\right) .
$$

Note that $n^{M} n_{M}=0$ (it is null) and $n^{M}$ satisfies the affine condition $n^{N} \nabla_{N} n^{M}=0$, even for the anisotropic background considered here.

The null Raychaudhuri equation takes the form

$$
\frac{d \hat{\theta}}{d \lambda}=\frac{-\hat{\theta}^{2}}{D-2}-|\hat{\sigma}|^{2}-R_{M N} n^{M} n^{N}
$$

Our corrections due to the Gauss-Bonnet term appear on the right hand side, as

$$
\begin{aligned}
& \frac{8}{\alpha^{\prime}} R_{M N} n^{M} n^{N} \sim H_{M N} n^{M} n^{N}=H_{t t} n^{t} n^{t}+H_{r r} n^{r} n^{r} \\
& =\frac{(d-1)(d-2)(d-3)(d-5)}{2} \frac{\dot{a}^{2}}{a^{6}}+2(d-1)(d-2)(d-3) \frac{\ddot{a} \dot{a}^{2}}{a^{5}} \\
& +\frac{(m-1)(m-2)(m-3)(m-5)}{2} \frac{\dot{b}^{4}}{a^{2} b^{4}}+2 m(m-1)(m-2) \frac{\ddot{b} \dot{b}^{2}}{a^{2} b^{3}}+2 m(d-1)(d-2) \frac{\dot{a}^{2}}{a^{4}} \frac{\ddot{b}}{b} \\
& +2 m(m-1)(d-1) \frac{\dot{b}^{2}}{b^{2}}\left[\frac{\ddot{a}}{a^{3}}-\frac{\dot{a}^{2}}{a^{4}}\right]+2(m-1) \tilde{R}_{m} \frac{\ddot{b}}{a^{2} b^{5}} .
\end{aligned}
$$

As before, choosing $d=3, m=1,2$ and a flat internal space $\tilde{R}_{m}=0$ simplifies the result considerably

$$
\begin{aligned}
H_{M N} n^{M} n^{N} & =2 m(m-1)(d-1) \frac{\dot{b}^{2}}{b^{2}}\left[\frac{\ddot{a}}{a^{3}}-\frac{\dot{a}^{2}}{a^{4}}\right]+2 m(d-1)(d-2) \frac{\dot{a}^{2}}{a^{4}} \frac{\ddot{b}}{b} \\
& =4 m(m-1) \frac{\dot{b}^{2}}{b^{2}} \frac{\dot{H}_{d}}{a^{2}}+4 m \frac{\dot{a}^{2}}{a^{4}} \frac{\ddot{b}}{b}
\end{aligned}
$$


where we wrote $H_{d} \equiv \dot{a} / a$, and thus $\dot{H}_{d}=\ddot{a} / a-\dot{a}^{2} / a^{2}$. The overall sign of this correction term is not clear; typically we expect $\dot{H}_{d} \leq 0$ for spacetimes that don't have NEC violating matter, so this term is potentially negative. However, it is unclear the scenarios for which $\ddot{b} / b<0$.

Finally, consider a comoving, proper-time parameterized timelike geodesic described by the tangent vector

$$
u^{M}=(1,0, \overrightarrow{0}, \overrightarrow{0}) \text {. }
$$

It is straightforward to check that $u^{M} u_{M}=-1$ and $u^{N} \nabla_{N} u^{M}=0$.

The timelike Raychaudhuri equation takes the form

$$
\frac{d \theta}{d \tau}=-\frac{\theta^{2}}{D-1}-|\sigma|^{2}-R_{M N} u^{M} u^{N},
$$

where again the corrections to the Raychaudhuri equation due to the Gauss-Bonnet corrections come from the last term

$$
\begin{aligned}
& \frac{8}{\alpha^{\prime}} R_{M N} u^{M} u^{N} \sim H_{M N} u^{M} u^{N}=H_{t t} u^{t} u^{t} \\
& =-\frac{d(d-1)(d-2)(d-3)}{2} \frac{\dot{a}^{4}}{a^{4}}-\frac{m(m-1)(m-2)(m-3)}{2} \frac{\dot{b}^{4}}{b^{4}}-d m(d-1)(m-1) \frac{\dot{a}^{2}}{a^{2}} \frac{\dot{b}^{2}}{b^{2}} \\
& +\frac{\tilde{R}_{m}}{b^{2}}\left[-d(d-1) \frac{\dot{a}^{2}}{a^{2}}+3 m(m-1) \frac{\dot{b}^{2}}{b^{2}}+\frac{3}{2} \frac{\tilde{R}_{m}}{b^{2}}\right] .
\end{aligned}
$$

Notice here that for a flat internal space $\tilde{R}_{m}=0$, these corrections are manifestly negative, thus they cause divergence in the Raychaudhuri equation.

More generally, we see that whether these additional correction terms due to Gauss-Bonnet act to increase or oppose convergence depends on the particular background, and whether one is considering timelike or null rays. Thus, there are backgrounds in which the usual singularity theorems no longer hold.

\section{Appendix C: Weyl Curvature Tensor}

In Section III, we encountered corrections to the induced 4-dimensional Einstein equations that involved the 5dimensional Weyl curvature through the tensor

$$
E_{\mu \nu}={ }^{(5)} C_{\mu \alpha \nu \beta} n^{\alpha} n^{\beta} .
$$

In this Appendix, we collect some useful properties of the Weyl tensor, as well as evaluate this term for the braneworld metric $(59)$.

\section{Weyl Tensor}

We begin with some facts about the Weyl curvature tensor. First, the definition of the Weyl curvature tensor is

$$
C_{\alpha \beta \gamma \delta}=R_{\alpha \beta \gamma \delta}+\frac{1}{n-2}\left[g_{\alpha \delta} R_{\gamma \beta}+g_{\beta \gamma} R_{\delta \alpha}-g_{\alpha \gamma} R_{\delta \beta}-g_{\beta \delta} R_{\gamma \alpha}\right]+\frac{1}{(n-1)(n-2)}\left[g_{\alpha \gamma} g_{\delta \beta}-g_{\alpha \delta} g_{\gamma \beta}\right] R
$$

in terms of the Riemann tensor, Ricci tensor, and Ricci scalar. In effect, the Weyl tensor $C_{\alpha \beta \gamma \delta}$ is the traceless part of the Riemann curvature tensor. As such, it inherits the usual symmetry identities from the Riemann tensor, as well as a traceless condition:

$$
\begin{aligned}
& C_{\alpha \beta \gamma \delta}=-C_{\alpha \beta \delta \gamma}=-C_{\beta \alpha \gamma \delta}=C_{\gamma \delta \alpha \beta} \\
& C_{\beta \alpha \delta}^{\alpha}=0 \quad \text { (true for any } 2 \text { contracted indicies) }
\end{aligned}
$$

The Weyl curvature tensor has an interesting feature: for any 2 metrics that can be related by a conformal factor

$$
\hat{g}_{\alpha \beta}=\Omega^{2} g_{\alpha \beta},
$$


the Weyl curvature tensors are the same

$$
\hat{C}_{\alpha \beta \gamma \delta}=C_{\alpha \beta \gamma \delta}
$$

This means that if a metric can be written in a conformally flat form, then $C_{\alpha \beta \gamma \delta}=0$ identically.

In particular, for the RS Minkowksi metric (61), this can be written in conformally flat form (with the coordinate redefinition of $\left.d \chi=e^{-K(z)} d z\right)$. Further, the ansatz metric (59), is almost conformally flat, aside from the factor $b(t, y)$; thus, we expect the Weyl curvature tensor for 59 to be proportional to the time- and space-derivatives of $b(t, y)$ (as we subsequently find it to be).

\section{Evaluating $E_{\mu \nu}$}

In Section III we evaluated the $E_{\mu \nu}$ tensor in the limit $y \rightarrow 0$, finding that $E_{\mu \nu} \propto \mu \frac{b^{2}}{a^{2}}$, consequently the singular terms do not contribute. However, we need to examine the consistency of this result. In particular, the induced Einstein equations (63) are not closed, and must be supplemented by additional constraints [28]. The Bianchi identity for the induced Einstein equations (63) implies (assuming that the brane EM tensor $\tau_{\mu \nu}$ obeys EM conservation 78 )

$$
\hat{\nabla}^{\mu} E_{\mu \nu}=\kappa_{5}^{2} \hat{\nabla}^{\mu} \pi_{\mu \nu}
$$

where $\hat{\nabla}^{\mu}$ is the covariant derivative constructed with respect to the $4 \mathrm{~d} \hat{q}_{\mu \nu}$ induced metric. Thus, we will examine the consistency of our limiting procedure by verifying that our result for $E_{\mu \nu}$ is indeed a solution of the Bianchi identity (C7).

Starting on the right-hand side, we rewrite the Bianchi identity as $\hat{\nabla}^{\mu} \pi_{\mu \nu}=\hat{\nabla}_{\mu} \pi_{\nu}^{\mu}$, and using the suitably raised versions of the $\pi_{\nu}^{\mu}$ tensor, it is straightforward to compute

$$
\hat{\nabla}^{\mu} \pi_{\mu t}=-\frac{1}{6} \rho \dot{\rho}-\frac{1}{2} \frac{\dot{a}}{a} \rho(p+\rho)=-\frac{\rho}{6}\left(\dot{\rho}+3 \frac{\dot{a}}{a}(\rho+p)\right)=0,
$$

where we used energy conservation of the brane matter $(78)$ to set this entire term to zero. Similar results are obtained for the $\nu=r, \theta, \phi$ components of the Bianchi identity (C7).

However, for the left-hand side of the $\nu=t$ component of the Bianchi identity, we obtain

$$
\hat{\nabla}_{\mu} E_{t}^{\mu}=3 \frac{\dot{a}}{a} \frac{\mu}{a^{4}}\left(1-b^{2}\right) \text {. }
$$

The only way to satisfy the Bianchi identity (C7), then, is to require the dark radiation term to vanish, $\mu=0$. Similar results are obtained for the $\nu=r, \theta, \phi$ components.

[1] R. Penrose, Gravitational collapse and space-time singularities, Phys. Rev. Lett. 14 (1965) 57-59.

[2] S. W. Hawking and R. Penrose, The Singularities of gravitational collapse and cosmology, Proc. Roy. Soc. Lond. A314 (1970) 529-548.

[3] A. Raychaudhuri, Relativistic cosmology. 1., Phys. Rev. 98 (1955) 1123-1126.

[4] H. Friedrich and J. M. Stewart, Characteristic initial data and wave front singularities in general relativity, Proc. Roy. Soc. Lond. A385 (1983) 345-371.

[5] N. Arkani-Hamed, P. Creminelli, S. Mukohyama and M. Zaldarriaga, Ghost inflation, JCAP 0404 (2004) 001 hep-th/0312100.

[6] T. Kobayashi, M. Yamaguchi and J. Yokoyama, G-inflation: Inflation driven by the Galileon field, Phys. Rev. Lett. 105 (2010) 2313021008.0603 .

[7] B.-H. Lee, C. H. Lee, W. Lee, S. Nam and C. Park, Dynamics of false vacuum bubbles with the negative tension due to nonminimal coupling, Phys. Rev. D77 (2008) 063502 [0710.4599.

[8] B.-H. Lee, W. Lee and D.-h. Yeom, Dynamics of false vacuum bubbles in Brans-Dicke theory, JCAP 1101 (2011) 005 1006.3127 .

[9] L. H. Ford and T. A. Roman, Motion of inertial observers through negative energy, Phys. Rev. D48 (1993) $776-782$ gr-qc/9303038.

[10] A. Conroy, A. S. Koshelev and A. Mazumdar, Defocusing of Null Rays in Infinite Derivative Gravity, JCAP 1701 (2017), no. 01,017 1605.02080. 
[11] A. Conroy, A. S. Koshelev and A. Mazumdar, Geodesic completeness and homogeneity condition for cosmic inflation, Phys. Rev. D90 (2014), no. 12, 1235251408.6205.

[12] C. G. Callan, Jr., R. C. Myers and M. J. Perry, Black Holes in String Theory, Nucl. Phys. B311 (1989) $673-698$.

[13] B. Zwiebach, Curvature Squared Terms and String Theories, Phys. Lett. 156B (1985) 315-317.

[14] D. J. Gross and J. H. Sloan, The Quartic Effective Action for the Heterotic String, Nucl. Phys. B291 (1987) 41-89.

[15] S. Frolov, I. R. Klebanov and A. A. Tseytlin, String corrections to the holographic RG flow of supersymmetric SU(N) $x$ $S U(N+M)$ gauge theory, Nucl. Phys. B620 (2002) 84-108 hep-th/0108106.

[16] G. Niz and N. Turok, Stringy corrections to a time-dependent background solution of string and M-Theory, Phys. Rev. D75 (2007) 126004 0704.1727.

[17] C. Fairoos, A. Ghosh and S. Sarkar, Black Hole Entropy production and Transport coefficients, 1802.00177.

[18] C. Lanczos, A Remarkable property of the Riemann-Christoffel tensor in four dimensions, Annals Math. 39 (1938) 842-850.

[19] D. Lovelock, The Einstein tensor and its generalizations, J. Math. Phys. 12 (1971) 498-501.

[20] T. Padmanabhan and D. Kothawala, Lanczos-Lovelock models of gravity, Phys. Rept. 531 (2013) 115-171 1302.2151.

[21] D. G. Boulware and S. Deser, String Generated Gravity Models, Phys. Rev. Lett. 55 (1985) 2656.

[22] C. Charmousis, Higher order gravity theories and their black hole solutions, Lect. Notes Phys. 769 (2009) $299-346$ 0805.0568 .

[23] C. Garraffo and G. Giribet, The Lovelock Black Holes, Mod. Phys. Lett. A23 (2008) 1801-1818 0805.3575.

[24] S. Deser and B. Tekin, Energy in generic higher curvature gravity theories, Phys. Rev. D67 (2003) 084009 hep-th/0212292.

[25] P. Brax, C. van de Bruck and A.-C. Davis, Brane world cosmology, Rept. Prog. Phys. 67 (2004) 2183-2232 hep-th/0404011.

[26] P. Horava and E. Witten, Heterotic and type I string dynamics from eleven-dimensions, Nucl. Phys. B460 (1996) 506-524 hep-th/9510209.

[27] L. Randall and R. Sundrum, A Large mass hierarchy from a small extra dimension, Phys. Rev. Lett. 83 (1999) 3370-3373 hep-ph/9905221.

[28] T. Shiromizu, K.-i. Maeda and M. Sasaki, The Einstein equation on the 3-brane world, Phys. Rev. D62 (2000) 024012 gr-qc/9910076.

[29] S. Mukohyama, Brane world solutions, standard cosmology, and dark radiation, Phys. Lett. B473 (2000) $241-245$ hep-th/9911165.

[30] D. Ida, Brane world cosmology, JHEP 09 (2000) 014 gr-qc/9912002.

[31] K. Ichiki, M. Yahiro, T. Kajino, M. Orito and G. J. Mathews, Observational constraints on dark radiation in brane cosmology, Phys. Rev. D66 (2002) 043521 astro-ph/0203272.

[32] P. Binetruy, C. Deffayet, U. Ellwanger and D. Langlois, Brane cosmological evolution in a bulk with cosmological constant, Phys. Lett. B477 (2000) 285-291 hep-th/9910219.

[33] P. Binetruy, C. Deffayet and D. Langlois, Nonconventional cosmology from a brane universe, Nucl. Phys. B565 (2000) 269-287 hep-th/9905012.

[34] D. Langlois, Cosmology of brane - worlds, in Proceedings, 6th RESCEU International Symposium on Frontiers in Astroparticle Physics and Cosmology: Tokyo, Japan, 4-7 Nov 2003. 2004. astro-ph/0403579

[35] J. D. Barrow and R. Maartens, Kaluza-Klein anisotropy in the CMB, Phys. Lett. B532 (2002) $153-158$ gr-qc/0108073.

[36] K. S. Stelle, Renormalization of Higher Derivative Quantum Gravity, Phys. Rev. D16 (1977) 953-969.

[37] T. P. Sotiriou and V. Faraoni, $f(R)$ Theories Of Gravity, Rev. Mod. Phys. 82 (2010) 451-497 0805.1726.

[38] F. D. Albareti, J. A. R. Cembranos, A. de la Cruz-Dombriz and A. Dobado, On the non-attractive character of gravity in $f(R)$ theories, JCAP 1307 (2013) 009 1212.4781.

[39] C. S. Santos, J. Santos, S. Capozziello and J. S. Alcaniz, Strong energy condition and the repulsive character of $f(R)$ gravity, Gen. Rel. Grav. 49 (2017), no. 4, 501606.02212.

[40] S. Nojiri, S. D. Odintsov and V. K. Oikonomou, Modified Gravity Theories on a Nutshell: Inflation, Bounce and Late-time Evolution, Phys. Rept. 692 (2017) 1-104 1705.11098.

[41] A. Ashtekar, Singularity Resolution in Loop Quantum Cosmology: A Brief Overview, J. Phys. Conf. Ser. 189 (2009) 0120030812.4703 .

[42] P. Singh, Are loop quantum cosmos never singular?, Class. Quant. Grav. 26 (2009) 125005 0901.2750.

[43] L.-F. Li and J.-Y. Zhu, Thermodynamics in Loop Quantum Cosmology, Adv. High Energy Phys. 2009 (2009) 905705 0812.3544 .

[44] A. K. Raychaudhuri, Theoretical Cosmology. Oxford, 1980.

[45] S. S. Haque and B. Underwood, Consistent Cosmic Bubble Embeddings, Phys. Rev. D95 (2017), no. 10, 103513 1701.07771 .

[46] S. Das, Quantum Raychaudhuri equation, Phys. Rev. D89 (2014), no. 8, 084068 1311.6539.

[47] S. Alsaleh, L. Alasfar, M. Faizal and A. F. Ali, Quantum no-singularity theorem from geometric flows, 1705.00977

[48] R. Wald, General Relativity. The University of Chicago Press, 1984. 